\title{
Effects of inflammatory factors including plasma tumor necrosis factor- $\alpha$ in the clinical treatment of acute respiratory distress syndrome
}

\author{
SHU RAN HUANG ${ }^{1,2}$, AI YING MA ${ }^{2}$, YUAN LIU ${ }^{1}$ and YAN QU ${ }^{1}$ \\ ${ }^{1}$ Department of Intensive Care Unit, The Affiliated Qingdao Municipal Hospital of Qingdao University, \\ Qingdao, Shandong 266071; ${ }^{2}$ Department of Intensive Care Unit, The Affiliated Hospital of \\ Jining Medical University, Jining, Shandong 272029, P.R. China
}

Received November 1, 2016; Accepted February 7, 2017

DOI: $10.3892 / \mathrm{ol} .2017 .6090$

\begin{abstract}
The present study investigated the association between the levels of plasma tumor necrosis factor- $\alpha$ (TNF- $\alpha$ ), interleukin (IL)-6, IL-8, high-sensitivity C-reactive protein and fibrinogen levels of patients with acute respiratory distress syndrome (ARDS) with ARDS treatment outcome and duration of mechanical ventilation, with the aim of improving the efficacy of ARDS therapy. A total of 140 patients with ARDS were randomly divided into groups $\mathrm{A}$ and $\mathrm{B}$, with 70 patients in each group. The patients in group A received a combination of conventional treatment and comprehensive treatment (test group) and the patients in group B were treated with conventional therapy only (control group). Elbow venous blood was obtained from each patient in the morning prior to treatment, on the 3rd day of treatment and on the 5th day of treatment. The levels of inflammatory factors, the clinical effects and the duration of mechanical ventilation of the two groups were statistically analyzed. The levels of IL-6, TNF- $\alpha$ and IL- 8 in patients from group A were significantly reduced compared with those from group $\mathrm{B}(\mathrm{P}<0.05)$. Additionally, the oxygenation index and arterial partial oxygen pressure of patients in group A were significantly higher compared with group B patients $(\mathrm{P}<0.05)$, the duration of mechanical ventilation of group A was decreased compared with that of group B $(\mathrm{P}<0.05)$ and the overall response rate of group A was $>90 \%$, whereas group B had a response rate of $80.0 \%$. These results indicate that the treatment administered to patients in group A exhibited an improved clinical efficacy. The combination of comprehensive and conventional therapy may effectively reduce the levels of inflammatory factors and the inflammatory response, and these levels may be important for the
\end{abstract}

Correspondence to: Professor Yan Qu, Department of Intensive Care Unit, The Affiliated Qingdao Municipal Hospital of Qingdao University, 1 Jiaozhou Road, Qingdao, Shandong 266071, P.R. China E-mail: yanqu1@outlook.com

Key words: plasma tumor necrosis factor, interleukin, acute respiratory distress syndrome, clinical treatment sequence effective treatment of ARDS and in reducing treatment duration. Therefore the current study may improve upon current clinical practice.

\section{Introduction}

Acute respiratory distress syndrome (ARDS) is induced by pathological changes, including pulmonary edema, pulmonary atelectasis and hyaloid membrane formation, which may be caused by severe intrapulmonary or extrapulmonary diseases (1). The clinical characteristics of ARDS include polypnea, cyanosis of lips and fingertips, respiratory distress (a result of extreme anoxia) that cannot be relieved by conventional oxygen therapy, chest distress, a cough and the presence of blood in the sputum (2). The pathogenesis of ARDS is complex and remains to be thoroughly clarified. Wang et al (3) hypothesized that the imbalance between inflammation and anti-inflammatory responses serves an important role in the development of ARDS. Ma et al (4) found that there were $>100$ factors associated with ARDS development. Tumor necrosis factor- $\alpha$ (TNF- $\alpha)$, interleukin (IL)-6, IL-8, high-sensitivity C-reactive protein (hs-CRP) and fibrinogen (FIB) are associated with inflammation and may be important in the treatment of ARDS as they are regulatory mediators of the inflammatory reaction (5).

TNF- $\alpha$, secreted by activated neutrophile granulocytes and mononuclear macrophages, is a multi-functional polypeptide (6). It may stimulate inflammatory cells and produce toxic effects when associated with corresponding receptors; tissues eventually become severely damaged due to the increase in inflammatory factors, including IL-6 and IL-8 (7). The mortality rate of ARDS is up to $50 \%$ (8), therefore, identifying an effective treatment for ARDS is of great importance. Yuan et al (9) performed an intraperitoneal injection of tumor necrosis factor (TNFR)-Fc $24 \mathrm{~h}$ prior to the administration of lipopolysaccharide, and identified a local remission of inflammation and a reduced rate of apoptosis of cells in the lung.

In the present study, two groups of patients were treated. One group received the conventional oxygen and mechanical ventilation therapy; the second group received a comprehensive treatment, which combined the intravenous injection 
of propofol and nitroglycerin (to accelerate the remission of pulmonary edema) with the conventional treatment. The levels of IL-6, TNF- $\alpha$, hs-CRP, IL- 8 and FIB, and the duration of mechanical ventilation treatment between treatment groups were compared. It was concluded that the combination of comprehensive and conventional treatment of ARDS may improve treatment efficacy vs. conventional treatment alone.

\section{Materials and methods}

Patients. A total of 140 adult patients with ARDS who were admitted into the Affiliated Hospital of Jining Medical University, Jining, Shandong, China between January 2015 and January 2016 were randomly selected. Patients were enrolled if they had the following high-risk factors: Acute onset respiration frequency or respiration distress; hypoxemia, defined as arterial partial pressure of oxygen $\left(\mathrm{PaO}_{2}\right)$ of $<60 \mathrm{mmHg}$ or fraction of inspiration oxygen $\left(\mathrm{FiO}_{2}\right)$ of $\leq 200 \mathrm{mmHg}$; invasive shallows observed in both lungs using a chest X-ray; pulmonary artery wedge pressure of $\leq 18 \mathrm{mmHg}$. Patients that had cardiogenic pulmonary edema induced by other causes, had disorders in cardiac, hepatic and renal function, or had incomplete medical records were excluded.

The patients were allocated using a randomized method, as follows: a total of 140 pieces of paper were prepared. Half of them were marked with A, and half with B. They were folded and placed into a box. Each patient took a piece of paper from the box. The patients were grouped into a test group (A) and a control group (B) according to the piece of paper that was drawn. The average age of patients in groups A and B were $45 \pm 6$ and $47 \pm 5$ years, and the ratio of male to female patients was 39:31 and 37:33, respectively. The age and gender ratio of the two groups were not identified as significantly different $(\mathrm{P}>0.05)$. The test group received conventional treatment and comprehensive treatment and the control group was treated only with conventional therapy.

The present study was reviewed and approved by the Affiliated Qingdao Municipal Hospital of Qingdao University (no. As20151221). The patients and their family members were informed of all experimental procedures, and informed consent was obtained.

Reagents and instruments. The reagents required in the present study included propofol (Ruiqi Bio-Technology Co., Ltd., Shanghai, China), TNF- $\alpha$ radioimmunoassay kit (Furui Runze Co., Ltd., Beijing, China), IL-6 ELISA kit (Enzyme-linked Biotechnology Co., Ltd., Shanghai, China) and IL-8 ELISA kit (Enzyme-linked Biotechnology Co., Ltd.). Instruments included an Allegra X-15R centrifuge and an IMMAGE 800 protein analyser (Beckman Coulter, Inc., Brea, CA, USA), an RT-2202 automatic coagulometer (Touching Technology Co., Ltd., Shanghai, China) and an i-STAT 300 blood-gas analyzer (Liaicheng Trade Co., Ltd., Nanjing, China).

Treatmentmethods.Treatmentgroups A and B wereadministered conventional treatment, which included eliminating causes of the disease, treatment of primary diseases, oxygen therapy and mechanical ventilation (oxygen concentration, 50\%). If oxygen therapy was ineffective, it was replaced with invasive mechanical ventilation. Alongside conventional treatment, the test group (A) was also administered comprehensive treatment, which included an intravenous injection of $0.028 \mathrm{mg} /$ $\mathrm{kg}$ propofol (Ruiqi Bio-Technology Co., Ltd.) followed by intravenous pumping of $0.5 \mathrm{mg} /(\mathrm{kg} / \mathrm{h})$ propofol. The intake of injection liquid was controlled, in order to reduce the burden on the heart and lungs. Nitroglycerin was combined with between 250 and $500 \mathrm{ml}$ of $110 \%$ glucose injection to expand vessels, reduce pulmonary arterial pressure, relieve vascular resistance and accelerate the reduction of pulmonary edema. Nutritional supplements, including protein, were administered via nasal feeding.

Detection method. Blood was obtained via the elbow veins on the morning of the first treatment, on the 3rd day after the start of treatment and on the 5th day after the start of treatment. Inflammatory factors were detected according to the following protocols. Totally $8 \mathrm{ml}$ of venous blood was collected from each patient. Initially, $6 \mathrm{ml}$ of venous blood was centrifuged at $4^{\circ} \mathrm{C}$ at $700 \mathrm{x} g$ for $8 \mathrm{~min}$ using an Allegra ${ }^{\circledR} \mathrm{X}-15 \mathrm{R}$ centrifuge (Beckman Coulter, Co., Ltd., Brea, CA, USA). Serum was separated after 5 min of standing.

In total, $2 \mathrm{ml}$ of the blood was used for detecting TNF- $\alpha$ levels with a radioimmunoassay. The blood was transferred to a tube containing 10\% EDTA and mixed. Following incubation $(1 \mathrm{~h})$ at room temperature, it was centrifuged at 1,000 $\mathrm{x} g$ at $25^{\circ} \mathrm{C}$ for $5 \mathrm{~min}$ to separate plasma, then was detected with the radioimmunoassay kit.

IL-6 and IL- 8 levels were detected in a further $2 \mathrm{ml}$ of the blood. The procedure was as follows: i) dilutions of negative and positive control samples were added to Chlamydia pneumoniae antigen-coated micropores. ii) The samples were covered and incubated at $37^{\circ} \mathrm{C}$ with $100 \%$ humidity for $1 \mathrm{~h}$. iii) The micropores were washed with washing liquid several times. Horseradish peroxidase conjugate (dilution, 1:300) was added. iv) The incubation step was repeated, followed by further washing. v) Tetramethylbenzidine substrate was added and incubated at room temperature for $20 \mathrm{~min}$ followed by the addition of $100 \mu 1$ stop buffer. Absorbance was read at $450 \mathrm{~nm}$ and the level of each item was calculated.

The remaining $2 \mathrm{ml}$ of blood was used for detecting the level of hs-CRP with nephelometry. The procedure was as follows: initially, a series of diluted standards were used with the IMMAGE 800 to produce a standard curve; the blood (dilution, 1:20) was then added into the analyzer; the final detection result was automatically displayed.

Subsequently, $0.2 \mathrm{ml}$ of $0.25 \%$ sodium citrate (Ruiqi Bio-Technology Co., Ltd.) was added to another $2 \mathrm{ml}$ of blood. The mixture was centrifuged at $700 \times g$ at $4^{\circ} \mathrm{C}$ for $5 \mathrm{~min}$. FIB was detected with turbidimetry centrifugation at $251.55 \mathrm{xg}$ at room temperature for 10 min using an RT-2202 automated Coagulation Analyzer (Shanghai Any-Touch Technologies Co., Ltd., Shanghai, China).

Venous blood was analyzed using an i-STAT ${ }^{\circledR} 300$ Blood-Gas Analyzer [Liai Trade (Nanjing) Co., Ltd., China] to obtain the level of partial oxygen pressure $\left(\mathrm{PaO}_{2}\right)$. The oxygenation index was calculated using the value of $\mathrm{PaO}_{2} / \mathrm{FiO}_{2}$. Following the application of pressure support ventilation and synchronized intermittent mandatory ventilation, the ventilator was removed. If ventilation frequency was $<5 / \mathrm{min}$, patients were able to easily cough phlegm, and inhaled oxygen 
Table I. Levels of associated inflammatory factors.

\begin{tabular}{|c|c|c|c|c|c|}
\hline Groups & TNF- $\alpha(n g / l)$ & IL-6 (ng/l) & IL-8 (ng/l) & hs-CRP (mg/l) & FIB (mg/l) \\
\hline \multicolumn{6}{|l|}{ Test group } \\
\hline Prior to treatment & $19.52 \pm 2.61$ & $25.39 \pm 2.25$ & $17.95 \pm 2.92$ & $8.39 \pm 2.54$ & $7.35 \pm 1.08$ \\
\hline 3 days following the start of treatment & $24.18 \pm 2.91$ & $32.42 \pm 1.45$ & $21.56 \pm 1.89$ & $10.23 \pm 2.18$ & $11.52 \pm 1.14$ \\
\hline 5 days following the start of treatment & $6.61 \pm 1.74^{\mathrm{a}, \mathrm{b}}$ & $8.14 \pm 1.26^{\mathrm{a}, \mathrm{b}}$ & $7.57 \pm 1.17^{\mathrm{a}, \mathrm{b}}$ & $3.21 \pm 1.98^{\mathrm{a}, \mathrm{b}}$ & $3.02 \pm 1.15^{\mathrm{a}, \mathrm{b}}$ \\
\hline \multicolumn{6}{|l|}{ Control group } \\
\hline Prior to treatment & $18.69 \pm 1.59$ & $24.12 \pm 2.84$ & $17.88 \pm 2.46$ & $8.41 \pm 2.28$ & $7.44 \pm 1.19$ \\
\hline 3 days following the start of treatment & $30.79 \pm 3.59$ & $38.47 \pm 2.41$ & $30.77 \pm 2.97$ & $11.25 \pm 1.89$ & $12.08 \pm 1.45$ \\
\hline 5 days following the start of treatment & $12.47 \pm 2.77$ & $16.33 \pm 2.14$ & $15.68 \pm 1.74$ & $5.47 \pm 1.42$ & $4.15 \pm 1.27$ \\
\hline
\end{tabular}

Data included are mean \pm standard deviation. ${ }^{\mathrm{a}} \mathrm{P}<0.05$ compared with prior to treatment. ${ }^{\mathrm{b}} \mathrm{P}<0.05$ compared with the control group. TNF- $\alpha$, tumor necrosis factor $\alpha$; IL, interleukin; hs-CRP, high-sensitivity C-reactive protein; FIB, fibrinogen.

Table II. Blood gas results of the two treatment groups.

\begin{tabular}{lcc}
\hline Groups & $\mathrm{PaO}_{2}(\mathrm{mmHg})$ & $\mathrm{PaO}_{2} / \mathrm{FiO}_{2}\left(\mathrm{mmHg}^{2}\right.$ \\
\hline Test group & & $123.84 \pm 17.48$ \\
Prior to treatment & $44.39 \pm 1.89$ & $236.69 \pm 14.28^{\mathrm{a}}$ \\
3 days following the start of treatment & $74.59 \pm 2.84^{\mathrm{a}}$ & $329.12 \pm 19.98^{\mathrm{a}}$ \\
5 days following the start of treatment & $96.21 \pm 2.17^{\mathrm{a}}$ & $125.50 \pm 18.73$ \\
Control group & & $201.37 \pm 14.24$ \\
Prior to treatment & $45.29 \pm 3.15$ & $271.00 \pm 13.85$ \\
3 days following the start of treatment & $66.79 \pm 3.57$ & $85.14 \pm 2.14$ \\
5 days following the start of treatment & & 27.00 \\
\hline
\end{tabular}

Data included are mean \pm standard deviation. ${ }^{\mathrm{a}}<0.05$ compared with prior to treatment. $\mathrm{PaO}_{2}$, arterial partial pressure of oxygen; FiO ${ }_{2}$, fraction of inspiration oxygen.

concentration was therefore $<50 \%$. The duration of mechanical respiration was recorded for each patient.

The levels of TNF- $\alpha$, IL-6, IL-8, hs-CRP, FIB and oxygenation index were measured and recorded before the experiment, 3 days after the experiment and 5 days after the experiment. The starting and ending times of mechanical respiration were recorded to calculate the duration of mechanical respiration.

Evaluation criteria of treatment efficacy. The treatment was considered to be clinically 'significantly effective' if pulmonary moist rales had subsided, breath sound and blood gas recovered to a normal level within 14 days of treatment and pulmonary shadows had been absorbed as observed using X-ray films after 10 days, as per previously described criteria (10). The treatment was considered to be 'effective' if the majority of the moist pulmonary rales had subsided, blood gas and breath sound recovered to normal within 7 days of initial treatment, and pulmonary shadows were improved as observed by X-ray films 10 days after initial treatment. Treatment was determined as 'ineffective' if moist pulmonary rales, blood gas, breath sound and pulmonary shadows did not indicate any improvement after 10 days of treatment.
Statistical analysis. The experimental data was analyzed using SPSS (version 17.0; SPSS, Inc., Chicago, IL, USA). Data are expressed as the mean \pm standard deviation. Enumerated data were processed by chi-square test. $\mathrm{P}<0.05$ was considered to indicate a statistically significant difference.

\section{Results}

Levels of inflammatory factors. The data in Table I demonstrates that 5 days after the start of treatment, the levels of hs-CRP, TNF- $\alpha$, IL- 6 and IL- 8 of the test group were significantly altered compared with the levels prior to treatment $(\mathrm{P}<0.05)$ and compared with those in the control group $(\mathrm{P}<0.05)$. The levels of inflammatory factors in the control group prior to and following treatment were not significantly different $(\mathrm{P}>0.05)$. The levels of the inflammatory factors hs-CRP, TNF- $\alpha$, IL- 6 and IL- 8 increased after the start of treatment but during the later stages of treatment, the levels of these factors decreased below the levels recorded prior to treatment.

Blood gas analysis. The data in Table II indicates that at 3 and 5 days after the start of treatment, the oxygenation index and $\mathrm{PaO}_{2}$ of the test group were significantly different compared 


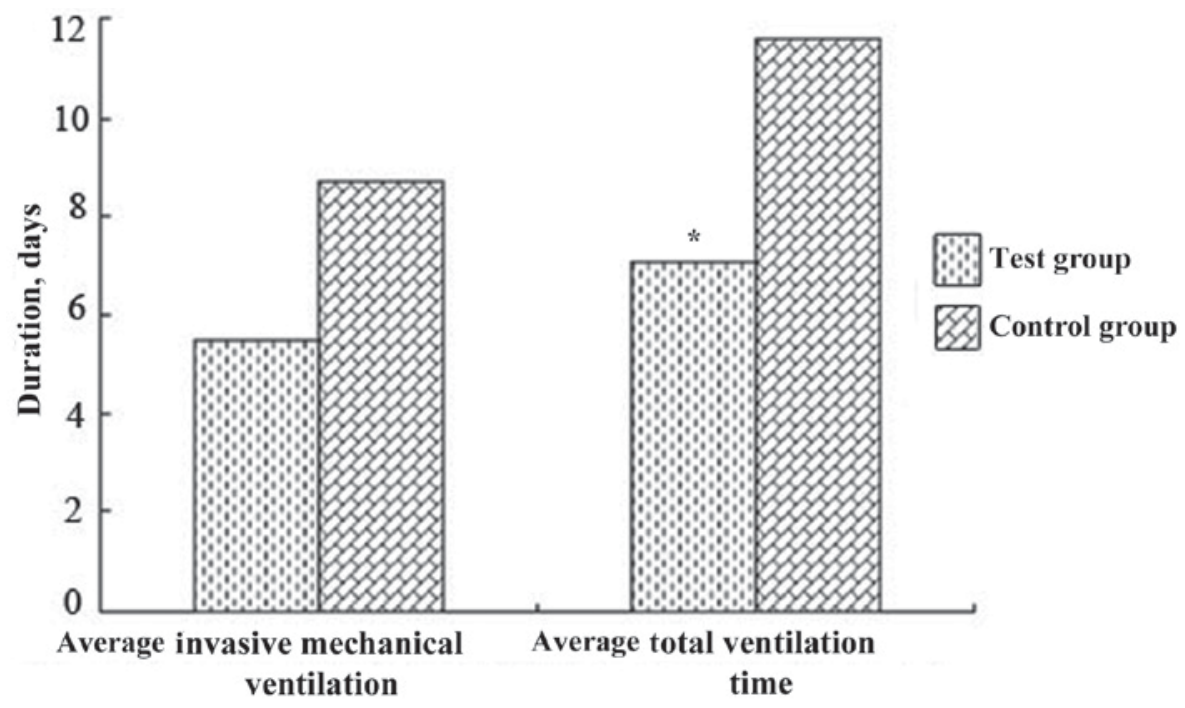

Figure 1. Mean overall duration of the mechanical ventilation in the test $(n=70)$ and control group $(n=70)$, including the time on invasive mechanical ventilation. "P $<0.05$ compared with the control group.

A

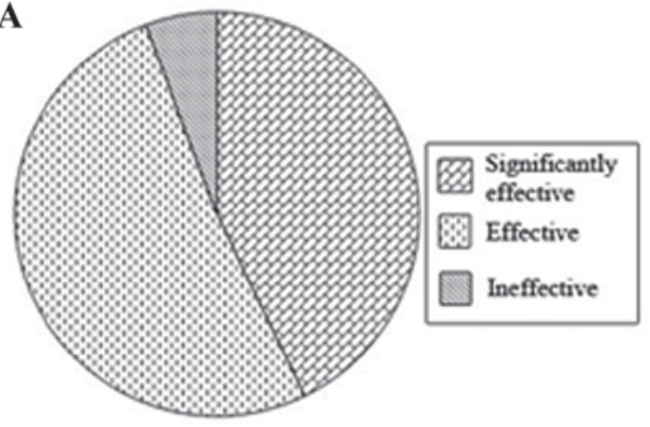

B

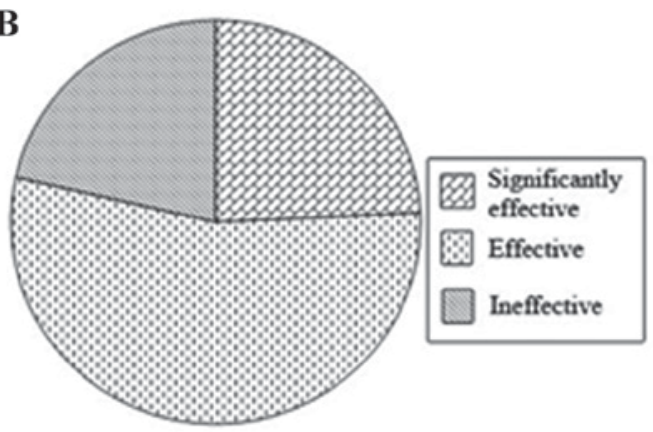

Figure 2. Clinical efficacy of acute respiratory distress syndrome treatment in (A) the test group and (B) the control group. The patient response to treatment indicated if the treatment was ineffective, effective or significantly effective. Significantly effective was determined by $\mathrm{P}<0.05$.

with those prior to treatment $(\mathrm{P}<0.05)$. However, the levels of these two factors were not significantly different $(\mathrm{P}>0.05)$ when compared between the test group and the control group. The oxygenation index and $\mathrm{PaO}_{2}$ of the control group prior to the start of treatment and at 3 and 5 days after the start of treatment were also not significantly different $(\mathrm{P}>0.05)$.

Duration of mechanical ventilation. Fig. 1 demonstrates that the mean duration of invasive ventilation and the mean overall duration of ventilation of the test group was decreased compared with that of the control group, and the difference in overall ventilation duration between the two groups was significantly different $(\mathrm{P}<0.05)$. This indicated that comprehensive treatment may reduce the duration of ventilation required and therefore ventilation weaning was improved and faster.

Clinical efficacy. Fig. 2 indicates that the test group had a higher number of patients that were administered effective treatment compared with the control group. The percentage of patients that demonstrated disease remission in the test group, including patients that received significantly effective and effective treatment, was $>90 \%$, whereas the percentage of patients in the control group with effective and significantly effective treatment was $\sim 80 \%$. Therefore, the number of the patients that responded well to treatment in the test group was higher compared with that of the control group.

\section{Discussion}

Ordinary infection, burns and septicemia are factors with a high risk of inducing ARDS, and this disease may be fatal if patients are not treated with highly effective therapy (11). A survey indicated that the mortality rate of ARDS may be up to $50 \%$ (12) and therefore, the timely administration of effective treatment is important to reduce ARDS-associated fatalities. In the current study, the levels of associated inflammatory factors were decreased in the test group, indicating that the combination of the comprehensive and conventional treatment administered to the test group affected the levels of the inflammatory factors. Additionally, in the test group the oxygenation index and $\mathrm{PaO}_{2}$ levels improved and the symptoms of oxygen deficiency and hypoxemia were alleviated. The reduced length of time patients received mechanical ventilation in the test group was beneficial for weaning patients off the ventilator. The overall response rate to therapy of the test group was $>90 \%$, which suggests that this treatment method was advantageous compared with the treatment of the control group and 
comprehensive and conventional combined treatment may significantly promote the relief of respiratory symptoms of ARDS and control the quantity of inflammatory fluids that accumulate in the lung.

ARDS may induce intense inflammatory reactions in the pulmonary interstitium as endogenous and exogenous irritants promote inflammation via stimulating monocytes and macrophages to express inflammatory factors, such as TNF and IL (13). According to the literature, inflammatory factors including hs-CRP, IL-8, TNF- $\alpha$, IL- 6 and FIB may be associated with the development of ARDS (14). TNF- $\alpha$ has been established to have roles in regulating immunity and the inflammatory response (15). High levels of TNF- $\alpha$ are associated with an increased risk of impaired and dysfunctional endothelial cells (16). IL-6 and IL-8 also have important roles in the inflammatory response, as they stimulate the release of cytase and promote the activity of platelet-activating factors (17). hs-CRP is a type of acute time phase reactive protein generated when the levels of certain proteins in plasma significantly increase or decrease, including during acute time phase reaction. It is one of the representative markers in vascular inflammation, and its level is associated with organ damage (18). The aforementioned inflammatory factors alter the response of monocytes and macrophages, promoting the release of inflammatory mediators that may result in damage to the lung tissue (19). Propofol inhibits the inflammatory response by reducing the activity of neutrophils (20). Notably, propofol effectively relieves the oxidative stress that is induced by acute pulmonary injury (21). For patients with ARDS, mechanical ventilation is an important therapeutic procedure that relieves dyspnea and hypoxemia symptoms and reduces pulmonary injury. The addition of appropriate nutritional supplements during treatment may promote normal metabolism and reduce overall nutritional loss (22).

In conclusion, the combination of conventional and comprehensive treatment of ARDS in the present study improves the oxygenation index and $\mathrm{PaO}_{2}$ level, reduces the levels of important inflammatory factors and relieves the inflammatory response, as well as improving respiratory symptoms and reducing pulmonary injury. Therefore, this combination therapy may be able to improve the therapeutic outcomes for patients with ARDs compared with the current clinical practice.

\section{References}

1. Komiya K, Ishii H, Murakami J, Yamamoto H, Okada F, Satoh K, Takahashi O, Tobino K, Ichikado K, Johkoh T and Kadota J: Comparison of chest computed tomography features in the acute phase of cardiogenic pulmonary edema and acute respiratory distress syndrome on arrival at the emergency department. J Thorac Imaging 28: 322-328, 2013.

2. Cardinale L, Volpicelli G, Lamorte A and Martino J; Andrea Veltri: Revisiting signs, strengths and weaknesses of standard chest radiography in patients of acute dyspnea in the emergency department. J Thorac Dis 4: 398-407, 2012.
3. Wang HY: The role of cytokines and inflammatory mediators in the pathogenesis of acute respiratory distress syndrome. Chin J Lung Dis: Electron Edit 7: 83-86, 2014.

4. Ma LJ and Li WP: Jin luminescence acute lung injury/acute respiratory distress syndrome: Research progress in the pathogenesis of the disease. Chin J Lung Dis: Electron Edit 6: 65-68, 2013.

5. Tangvarasittichai $\mathrm{S}$, Pongthaisong $\mathrm{S}$ and Tangvarasittichai $\mathrm{O}$ : Tumor necrosis factor-A, interleukin-6, C-reactive protein levels and insulin resistance associated with type 2 diabetes in abdominal obesity women. Indian J Clin Biochem 31: 68-74, 2016.

6. Kim S, Yamamoto K, Nakamura Y, Otoyo Y and Yamatodani A: A possible mechanism of cisplatin-induced tumor necrosis factor (TNF)- $\alpha$ production in murine macrophages. Pharmacol Pharm 4: 146-151, 2013.

7. Yan X, Wang XY, Sheng YH, Zhu L, Zhang LD and Zang Q: Exploration of the theory of 'Fei and Dachang being interior-exteriorly related' from observing changes of inflammatory cytokines and oxygen free radicals in the lung tissue of ulcerative colitis rats. Zhongguo Zhong Xi Yi Jie He Za Zhi 34: 455-459, 2014 (In Chinese).

8. Wang JL and Hu JL: New progress of respiratory support therapy for treating acute respiratory distress syndrome. J Clin Pulmon Med: 533-536, 2016.

9. Yuan WF, Li L, Li YF, et al: Effects of TNF- $\alpha$ on lung tissue apoptosis in mice with acute lung injury induced by lipopolysaccharide. Int J Respirat 32: 565-568, 2012.

10. Hudord WE, Bigatello LM, Haspel KL, Hess DR and Warren RL (eds): In: Critical care handbook of the massachusetts general hospital.3rd edition. Shenyang: Liaoning Science and Technology Press: 257-267, 2001.

11. Bein T, Grasso S, Moerer O, Quintel M, Guerin C, Deja M, Brondani A and Mehta S: The standard of care of patients with ARDS: Ventilatory settings and rescue therapies for refractory hypoxemia. Intensive Care Med 42: 699-711, 2016.

12. Liang JY, Jia JF and Peng NG: Clinical analysis of acute respiratory distress syndrome caused by blunt chest trauma. Chin J Tradit Chin Med 02: 139-140, 2010.

13. Huang YZ and Qiu HB: Current situation and challenge of mechanical ventilation strategy for acute respiratory distress syndrome. Intern Med Theory Pract 05: 470-475, 2010.

14. Yang LJ: Serum levels of IL-6, IL-8, TNF-, hs-CRP and lung function in patients with moderate to severe chronic obstructive pulmonary disease. J Clin Pulmon: 481-484, 2016.

15. Scharl M: Pathophysiological role of TNF in inflammatory bowel disease: TNF and its effect on innate immune defense. Front Gastroint Res 34: 49-55, 2015.

16. Sun ZJ, Lu Q and Li YP: Cytokines and inflammatory mediators in acute respiratory distress syndrome. Zhongguo Wei Zhong Bing Ji Jiu Yi Xue 15: 186-189, 2003 (In Chinese).

17. Roux J, Mcnicholas CM, Carles M, Goolaerts A, Houseman BT, Dickinson DA, Iles KE, Ware LB, Matthay MA and Pittet JF: IL-8 inhibits cAMP-stimulated alveolar epithelial fluid transport via a GRK2/PI3K-dependent mechanism. FASEB J 27: 1095-1106, 2013.

18. Wang CY, Wu H, Wang W, et al: Significance of PCT, HS-CRP and FIB in the treatment of pulmonary tuberculosis. J Clin Pulmon: 1608-1610, 2015 .

19. Yang Q: Acute lung injury and inflammatory mediators. Clin Pulmon Med J 16: 741-743, 2011.

20. Yang T and Lu JX: Experimental study on the protective effect of propofol on severe acute pancreatitis associated lung injury. Zhejiang Depart Traum Surg 17: 312-314, 2012.

21. Wang X, Liu C and Wang G: Propofol protects rats and human alveolar epithelial cells against lipopolysaccharide-induced acute lung injury via inhibiting HMGB1 expression. Inflammation 39: 1004-1016, 2016.

22. Lee BC, Kyoung KH, Kim YH and Hong SK: Non-invasive ventilation for surgical patients with acute respiratory failure. J Korean Surg Soc 80: 390-396, 2011. 